Internist 2010 $51: 127-128$

DOI 10.1007/s00108-009-2530-8

Online publiziert: 4. Dezember 2009

(c) Springer-Verlag 2009

\author{
A. Widmer ${ }^{1} \cdot$ P. Gastmeier ${ }^{2} \cdot$ M. Battegay ${ }^{1}$ \\ ${ }^{1}$ Klinik für Infektiologie \& Spitalhygiene, Universitätsspital, Basel \\ 2 Institut für Hygiene und Umweltmedizin, Charité Universitätsmedizin Berlin
}

\title{
Infektprävention im Fokus
}

Die Krankenhaushygiene beinhaltet schwerpunktmäßig die Prävention im Krankenhausbereich erworbener, d. h. nosokomialer, Infektionen, die Verhinderung der Verbreitung von multiresistenten Erregern sowie das Epidemiemanagement.

\section{- Ein Krankenhausbetrieb ohne Krankenhaushygiene und Infektprävention wäre heutzutage nicht denkbar.}

Viele der über die Jahrzehnte gewonnenen Einsichten und Errungenschaften tragen dazu bei, infektbedingte Morbidität und Mortalität auf einem möglichst tiefen Niveau zu halten. $\mathrm{Zu}$ den wichtigsten gehören allgemeine Vorsichtsmaßnahmen wie die Händehygiene, die Zuweisung in ein Einzelzimmer bei bestimmten resistenten Keimen oder der Schutz des Personals vor Erkrankungen mit speziellen Entsorgungssystemen für spitze Gegenstände oder gebrauchtes Injektionsmaterial. Septikämien gehören zu den gefährlichsten nosokomialen Infektionen und postoperative Wundinfektionen, neben den Harnwegsinfektionen, zu den häufigsten. Daher richten sich die Aktivitäten v. a. der Vermeidung von Katheterseptikämien und postoperativen Infektionen.

Die klassische Krankenhaushygiene widmete sich in der Vergangenheit v. a. der Exposition von gefährlichen Keimen. Im Fokus standen dabei Reinigung, Desinfektion und Sterilisation. Heute richtet sie sich auf die evidenzbasierte, klinisch messbare Reduktion nosokomialer Infek- tionen und weniger auf die Vermeidung einer Exposition, die nur möglicherweise zu einer Infektion führen könnte. Ein standardisiertes Prozedere widmet sich der Isolation von Patienten, z. B. bei Tuberkulose, oder dem Personalscreening auf methicillinresistente Staphylococcus aureus. Schließlich erfordern der Verdacht oder der Ausbruch einer Epidemie im Krankenhaus oder außerhalb des Krankenhauses spezielle koordinierte Maßnahmen, wie wir sie derzeit bei der $\mathrm{H}_{1} \mathrm{~N}_{1}$-Pandemie erleben.

In diesem Schwerpunktheft beabsichtigen wir, breit gefächert über aktuelle Gebiete der Krankenhaushygiene und Infektprävention evidenzbasiert zu berichten. In einem ersten übersichtlichen Artikel zeigt Gastmeier auf, welche Aspekte evidenzbasierter Leitlinien zu berücksichtigen sind. Als wichtiges Beispiel ist die Compliance zur Händedesinfektion, wohl die Basis aller Krankenhaushygiene, aufgezeigt.

In einem Artikel über die Übertragung von Problemkeimen im ambulanten Bereich schildern Luft und Dettenkofer, dass alte und neue häufiger auftretende multiresistente Erreger, z. B. MRSA (methicillinresistente Staphylococcus aureus), VRE (vancomycinresistente Enterokokken) und gramnegative Enterobakterien mit ESBL- (Extended-Spectrum Beta-Lactamase) Bildner eine zunehmende Herausforderung an Präventions- und Kontrollmaßnahmen stellen. Gerade in der Praxis sind multiresistente Erreger in den letzten Jahren deutlich häufiger aufgetreten.

Immer mehr beschäftigen postoperative Wundheilungsstörungen, die meist durch Infektionen bedingt sind, Internisten in der Ambulanz. Aufgrund verkürzter Hospitalisationszeiten, nach zuweilen komplexen chirurgischen Eingriffen, treten Wundinfektionen vermehrt nach Krankenhausaustritt auf. Wir schildern die wichtigsten pathophysiologischen Grundlagen, die notwendige Diagnostik und Therapie entsprechender postoperativer Komplikationen und prophylaktische Maßnahmen, um diese zu verhindern.

Spezifische Aspekte, welche im Krankenhausbereich häufig auftreten, dürfen bei einem solchen Schwerpunktheft nicht fehlen. Gerade die globale Zunahme der Clostridium-difficile-assoziierten Infektionen und das Auftreten so genannter hypervirulenter Stämme wird von Kola eingehend beschrieben. Diese aggressiveren Stämme erfordern striktere Hygienemaßnahmen beim Umgang von betroffenen Patienten.

\section{( ) Ängste im Zusammenhang mit Problem- und multiresistenten Keimen können durch fachgerechtes Wissen abgebaut werden}

Der letzte Artikel von Bühlmann und Widmer ist im Hinblick auf die saisonale Grippe und die $\mathrm{H}_{1} \mathrm{~N}_{1}$-Epidemie relevant. Infektionen der oberen Atemwege gehören zu den häufigsten Ursachen für Absenzen von der Schule oder Arbeit. Im Krankenhaus ist eine sorgfältige Hygiene mitunter mit Isolationsmaßnahmen bei respiratorischen Infektionen wichtig. Dies 
dient dem Schutz des Personals sowie der Verhinderung nosokomialer Infektionen im stationären und ambulanten Setting.

Wir hoffen, dass die zusammengestellten Artikel im Gebiet Krankenhaushygiene und Infektprävention einen Beitrag für die kontinuierliche Weiterbildung des Internisten zu leisten vermögen. Beabsichtigt ist, dass Ängste im Zusammenhang mit Problem- und multiresistenten Keimen durch fachgerechtes Wissen und interdisziplinäres Handeln abgebaut werden können. Gerade Patienten, die mit multiresistenten Erregern kolonisiert oder infiziert sind, sollen durch die präventiven Maßnahmen die selbe medizinische Versorgung erhalten wie alle anderen.

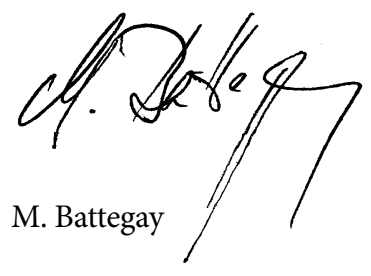

\section{Korrespondenzadresse}

\section{Prof. Dr. M. Battegay}

Klinik für Infektiologie \& Spitalhygiene, Universitätsspital Petersgraben 4, 4031 Basel Schweiz

mbattegay@uhbs.ch

\section{Springer Award Gefäßmedizin 2010}

\section{Jetzt einreichen! Brückenschlag von experimentellen Ergebnissen zum klinischen Nutzen wird ausgezeichnet}

Die Gefäßchirurgie ist ein Wachstumsfach mit zunehmender Bedeutung im Gesamtkonzept der Diagnostik und Therapie von Gefäßerkrankungen. Die wissenschaftliche Leitung der Gefässchirurgie aus Mitgliedern der deutschen, österreichischen und schweizerischen Gesellschaften für Gefäßchirurgie möchten daher zusammen mit dem Springer-Verlag die wissenschaftliche Forschung auf diesem Gebiet unterstützen und insbesondere den Nachwuchs durch die Verleihung des 1. Springer Award Gefäßmedizin fördern.

Nutzen Sie die Gelegenheit und reichen Sie Ihren Beitrag bis zum 01.06.2010 ein. Auf den Preisträger wartet ein Reisestipendium im Wert von 2500,00 EUR!

\section{Teilnahmebedingungen}

- Der Autor soll einen Übersichtsbeitrag in deutscher Sprache auf Grundlage einer eigenen experimentellen Arbeit verfassen. Bei der Beurteilung wird besonderer Wert auf den edukativen Charakter des Artikels sowie die Herausarbeitung der klinischen Relevanz gelegt - der Beitrag soll eine Brücke von den experimentellen Ergebnissen zur klinischen Anwendung schlagen.

- Es können eigene Originaldaten publiziert werden oder auch bereits publizierte eigene Originaldaten zusammengefasst und mit einem Ausblick zur klinischen Relevanz versehen werden. In letzterem Fall ist beim erstpublizierenden Verlag vor Manuskripteinreichung eine Genehmigung einzuholen.

- Das Teilnehmeralter ist auf 40 Jahre beschränkt.

- Der Beitrag darf eine Länge von max. 25.000 Zeichen (inkl. Leerzeichen) nicht überschreiten.
Alle eingereichten Beiträge werden durch eine Jury aus dem Editorial Board und dem Herausgebergremium unter der Leitung der Schriftleitung begutachtet.

\section{Preisverleihung bei der Dreiländertagung}

Die Verleihung des ersten Springer Award Gefäßmedizin erfolgt im Rahmen der

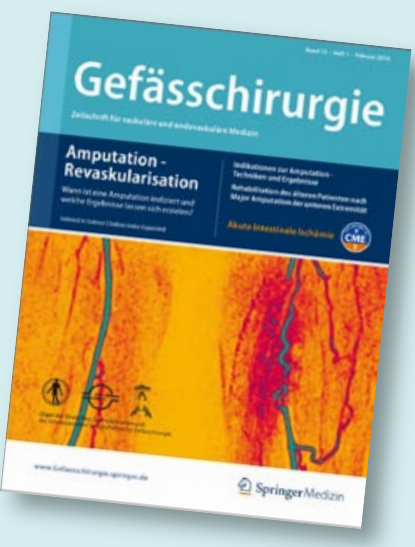

Dreiländertagung der deutschen, österreichischen und schweizerischen Gesellschaften für Gefäßchirurgie vom 7.-11. September 2010 in Berlin.

Vier der besten Arbeiten inklusive der Preisträger-Arbeit werden im Anschluss an die Dreiländertagung in Ausgabe 6/2010 in der Zeitschrift Gefässchirurgie veröffentlicht.

Bitte reichen Sie Ihren Beitrag zusammen mit Ihrem Lebenslauf in elektronischer Form bei der Verlagsredaktion ein: Tina.Suhai@springer.com

Teilnahmeschluss: 01.06.2010

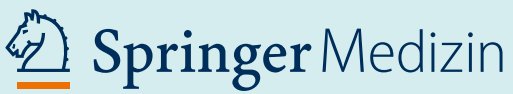

